Certificats d'incapacité de travail: les conventions cantonales sont gage de plus de sécurité

Le présent article se penche sur les différents pièges tendus par les certificats d'incapacité de travail. II décrit les points qui devraient nécessairement y figurer et plaide en faveur de certificats (plus) détaillés.

Dans différents cantons, les certificats d'incapacité de travail font I'objet de conventions entre les partenaires sociaux et les sociétés de médecine, une solution judicieuse tant aux yeux des partenaires sociaux que d'un point de vue juridique.

En juin 2007, la NZZ décrivait ainsi les avantages de tels certificats pour les relations entre les différentes parties en présence: «Peu après le début de la phase pilote dans la partie saintgalloise de la vallée du Rhin, on a constaté une amélioration de la communication entre les médecins et les employeurs, et un meilleur niveau de confiance mutuelle, explique Peter Bürki, secrétaire de l'Association patronale de la vallée du Rhin» [1].

Par ailleurs, au regard de la sécurité du droit, les conventions can- tonales vont aussi dans le sens des médecins. En effet, elles distinguent, à ma connaissance, deux types de certificats:

- un certificat d'incapacité de travail simple et gratuit pour les nombreux cas incontestés,

- et un certificat détaillé, expressément demandé par l'employeur et à sa charge, fondé sur une description du poste établie par l'employeur, que le médecin ne délivre qu'en accord avec le patient.

Ceci permet de répartir clairement les rôles. Le médecin peut en premier lieu établir un simple certificat, le moins coûteux pour tout le monde. II sait qu'aucun employeur ne pourra lui reprocher d'avoir ainsi voulu dissimuler une incapacité de travail problématique car l'employeur qui se verra remettre ce certificat pourra simplement commander un certificat détaillé.

Hanspeter Kuhn, avocat et secrétaire général adjoint de la FMH 1 Kosten sparen mit einem Zeugnis weniger - NZZ, 17. Juli 2007

\title{
Arbeitsunfähigkeitszeugnisse: Ärzte zunehmend im Fokus der Justiz (Teil 2)
}

\author{
Im ersten Teil dieses Beitrags, erschienen in der letzten SÄZ-Ausgabe, wurden an \\ einem Fallbeispiel die rechtlichen Grundlagen für die Erstellung ärztlicher Zeugnisse \\ dargestellt. Im folgenden werden Empfehlungen für die tägliche Praxis gegeben, die \\ es Ärzten ermöglichen sollen, sich vor rechtlichen Angriffen zu schützen.
}

Roger Rudolph

Korrespondenz:

Dr. iur. Roger Rudolph Streiff Pellegrini \& von Kaenel Rechtsanwälte Bahnhofstrasse 67 CH-8620 Wetzikon rudolph@streiffpartner.ch
Die schon seit längerem bei Arbeitgebern zu beobachtende Tendenz, ärztlichen Zeugnissen zu misstrauen, hat in den letzten Jahren eher noch zugenommen. Als Folge davon müssen sich Ärzte immer häufiger für die Qualität der von ihnen ausgestellten Zeugnisse verantworten, sei es gegenüber Vertrauensärzten, als Zeugen in einem arbeitsrechtlichen Zivilprozess oder gar als Angeschuldigte in einem Strafverfahren.

\section{Ausführlichere Zeugnisse schützen Arzt und Patienten}

\section{Ausgangslage}

Arbeitgeber misstrauen ärztlichen Zeugnissen vor allem dann, wenn sie von nur geringer inhaltlicher Aussagekraft sind. Es ist verständlich, dass Zeugnisse, die in einem einzigen Satz eine «Arbeitsunfähigkeit von $100 \%$ bis auf weiteres» bescheinigen, von Arbeitgebern kritisch hinterfragt werden. Erst recht gilt dies, wenn das Zeugnis im Umfeld einer sich abzeichnenden oder bereits erfolgten Kündigung ausgestellt wird. Denn in diesem Fall droht dem Arbeitge- ber nach den Regeln des Obligationenrechts eine mehrmonatige Verschiebung des Arbeitsvertragsendes, verbunden mit empfindlichen Lohnfortzahlungsfolgen [1]. Sehr häufig handelt es sich um psychisch oder mobbingbedingte Krankmeldungen. Wenn der Arbeitnehmer in einem solchen an sich schon von gegenseitigem Misstrauen geprägten Umfeld auch noch ein rudimentäres und häufig auch noch rückwirkendes ärztliches Attest vorlegt, muss man Verständnis dafür haben, dass der Arbeitgeber eine solche Krankmeldung nicht einfach ergeben abnickt.

\section{Minimaler Zeugnisinhalt}

Wie im ersten Teil in Heft 22 der SÄZ dargestellt, fehlen konkrete gesetzliche Vorgaben, wie ein ärztliches Zeugnis formuliert sein muss. Dennoch hat sich in der Praxis ein gewisser Mindeststandard herausgebildet. In diesem Zusammenhang sind als sinnvolle Ergänzung auch die in verschiedenen Kantonen praktizierten Vereinbarungen zwischen den Arbeitgeberverbänden und kantonalen Ärzteschaften zu erwähnen [2]. 


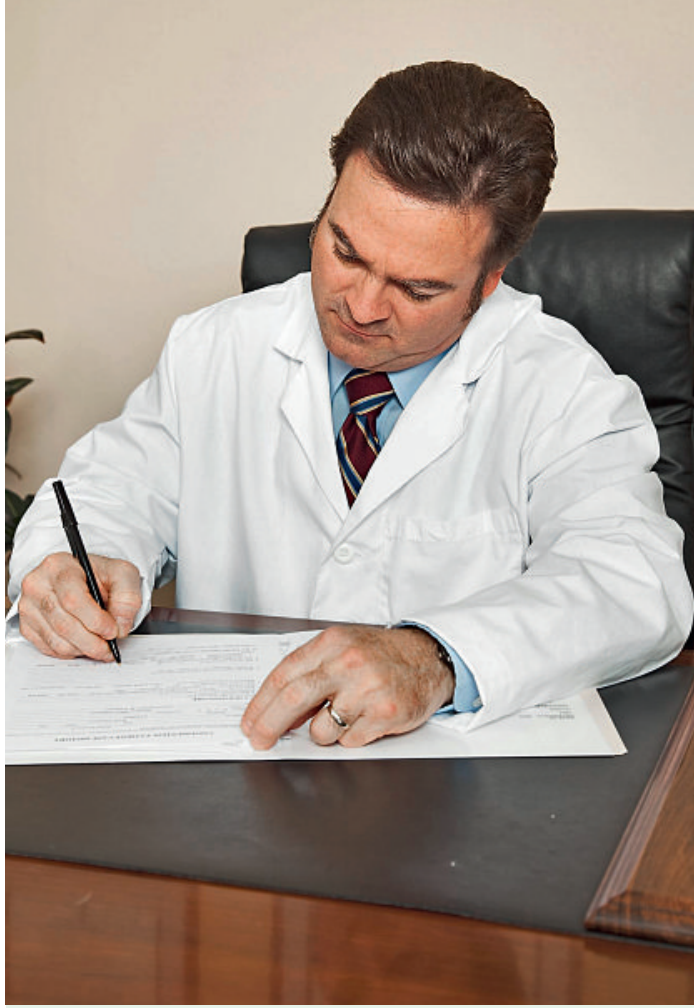

Sich Zeit zu nehmen für ein ausführlicheres Arbeitsunfähigkeitszeugnis, kann späteren Ärger vermeiden.

Selbstverständlich ist zunächst, dass der Arzt nur solche Tatsachen bescheinigen darf, die er nach seiner sorgfältigen und fachkundigen Einschätzung auch für wahr hält. Das heisst nicht, dass sich ein Arzt nie irren darf, denn das Irren allein begründet weder zivil- noch strafrechtlich eine Haftung. Erst wenn der Irrtum auf Fahrlässigkeit oder gar Vorsatz zurückzuführen ist, kann der Arzt zur Rechenschaft gezogen werden.

Zum minimalen Zeugnisinhalt zählen Beginn und Grad sowie voraussichtliche Dauer der Arbeitsunfähigkeit. Aus versicherungsrechtlichen Gründen ist weiter zwischen Krankheit und Unfall zu unterscheiden. Ausserdem sollen die Adresskoordinaten des Arztes ersichtlich sein (durch Stempel oder Brief-

\section{Erst wenn ein Irrtum auf Fahrlässigkeit oder gar Vorsatz zurückzuführen ist, kann der Arzt zur Rechenschaft} gezogen werden

papier) und ist das Zeugnis zu datieren und zu unterzeichnen. Nach Art. 34 der Standesordnung FMH ist schliesslich der Adressat des Zeugnisses zu nennen, also in der Regel der Arbeitgeber [3].

Zurückhaltung ist bei rückwirkenden Zeugnissen geboten, wenn also der Arzt den Beginn einer Arbeits-

\section{Certificats d'incapacité de travail:}

\section{les médecins toujours plus}

\section{dans la ligne de mire de la justice (2 ${ }^{\mathrm{e}}$ partie)}

Même s'il manque des dispositions légales concrètes, une norme minimale s'est établie dans la pratique pour la forme et le contenu des certificats. Elle consiste en la description du début, du degré et de la durée probable de l'incapacité de travail, aussi en la distinction entre maladie et accident. En outre, le médecin doit dater et signer le certificat, $y$ indiquer ses coordonnées et son titre de spécialiste. Ce contenu minimal n'est toutefois pas suffisant dans bien des cas, il peut être contreproductif pour le médecin et le patient ou même s'avérer dangereux s'il est toujours limité à un minimum. C'est particulièrement vrai pour les certificats établis rétroactivement ou qui ne peuvent pas être fondés sur des constatations objectives, comme c'est souvent le cas pour les maladies psychiques, l'épuisement professionnel ou le mobbing. Si un médecin ne peut s'appuyer que sur la description du patient et qu'il ne le mentionne pas dans le certificat, il doit s'attendre rapidement à des conséquences juridiques. De surcroît, des certificats plus explicites ont une plus grande force de persuasion face aux employeurs qui les acceptent bien mieux. Il est donc dans I'intérêt des médecins et des patients que les certificats médicaux contiennent davantage d'informations.

unfähigkeit für einen Termin vor der Erstkonsultation bescheinigen soll [4]. Das ist zwar als solches nicht unzulässig, zumal wenn der Patient bei der Erstkonsultation noch immer krank ist. Nach den Empfehlungen der Ärzte-Gesellschaft des Kantons Zürich (AGZ) soll es sich aber um Ausnahmefälle handeln und darf die Rückwirkung eine Woche nicht überschreiten [5]. In jedem Fall ist eine eigene Untersuchung unentbehrlich, denn eine Arbeitsunfähigkeitsbescheinigung, die ausschliesslich auf die Schilderung des Patienten abstellt, hat von vornherein keinen Beweiswert [6].

\section{Plädoyer für ausführlichere Zeugnisse}

Die arbeitsrechtliche Beratungspraxis zeigt, dass es sowohl für den Arzt wie für den Patienten bzw. Arbeitnehmer meistens von Vorteil wäre, wenn das ärztli- 
che Zeugnis gegenüber dem vorhin umrissenen Minimalinhalt einen etwas höheren Detaillierungsgrad aufweisen würde. Dies gilt jedenfalls für bestimmte typische Konstellationen, auf die gleich noch eingegangen wird. Mit einem höheren Detaillierungsgrad ist nicht die Diagnose gemeint, denn diese muss der Arbeitnehmer gegenüber seinem Arbeitgeber nicht gegen seinen Willen offenbaren [7]. Es geht vielmehr um ergänzende Informationen, welche die Modalitäten der Untersuchung näher umschreiben und deren proaktive Offenlegung die Glaubwürdigkeit des Zeugnisses erheblich steigern kann. Zu denken ist hier je nach konkreter Situation an folgendes:

- Chronologie der Behandlung: Nennung der Daten der Erstkonsultation, der weiteren bereits erfolgten Konsultationen sowie des nächsten bevorstehenden Termins (bzw. des Zeitpunkts der Neubeurteilung). Letzteres ist vor allem dann angezeigt, wenn eine Arbeitsunfähigkeit auf unbestimmte Dauer bescheinigt wird;

- Bei Teilarbeitsfähigkeit: Angabe, ob sich die reduzierte Arbeitsfähigkeit auf die Arbeitszeit oder die Arbeitsleistung bezieht und welche Arbeiten davon betroffen sind. Letzteres bedingt, dass sich der Arzt beim Arbeitnehmer oder - mit dessen $\mathrm{Zu}$ stimmung - beim Arbeitgeber über die Art der auszuführenden Arbeiten erkundigt [8].

- Nicht überprüfbare Angaben: Soweit dem Attest nicht überprüfbare Angaben des Patienten zugrunde liegen, sollte dies im Zeugnis wertungsfrei vermerkt werden. Es ist dies vielleicht die wichtigste Massnahme, die ein Arzt vorsorglich treffen kann, um sich selber zu schützen. Diese Empfehlung gilt neben objektiv kaum fassbaren psychischen Leiden, Burn-out oder Mobbing vor allem auch für rückwirkende Zeugnisse, wenn der Arzt aufgrund seiner eigenen Untersuchung rückwir-

\section{Mit Hilfe einer sorgfältig dokumentierten Kranken- geschichte kann der Vorwurf einer unsorgfältigen Untersuchung meist rasch entkräftet werden}

kend keine Arbeitsunfähigkeit mehr feststellen kann. Diesem Umstand kann der Arzt mit der Ergänzung «nach Angaben des Patienten» im Zeugnis Rechnung tragen, allenfalls präzisiert durch ein «ganz» oder «teilweise» [9]. Andernfalls droht ihm der nicht ganz von der Hand zu weisende Vorwurf, eine blosse - und möglicherweise falsche - Parteibehauptung des Arbeitnehmers durch seine ärztliche Autorität fahrlässig veredelt $\mathrm{zu}$ haben. Im schlimmsten Fall könnte bereits dies genügen, um ein Verfahren wegen falschen ärztlichen Zeugnisses ins Rollen zu bringen. Ge- nau dies ist in dem im ersten Teil des Beitrags (SÄZ 22/2010) geschilderten Fall dem Psychiater widerfahren, der sich deswegen vor Gericht verantworten musste [10]. Das Obergericht des Kantons Zürich hat ihn zwar vom Vorwurf des vorsätzlichen falschen Zeugnisses bzw. der Beihilfe zum Betrug freigesprochen. Das Gericht hat in seinen Erwägungen aber durchblicken lassen, dass eine Strafbarkeit wegen fahrlässigen falschen Zeugnisses durchaus denkbar gewesen wäre. Dass sich der Psychiater unter diesem Gesichtspunkt nicht verantworten musste, hing einzig mit einer prozessualen Nachlässigkeit der Staatsanwaltschaft zusammen, die es versäumt hatte, neben dem Vorsatz- auch den Fahrlässigkeitstatbestand rechtsgenügend anzuklagen.

- Bei arbeitsplatzbezogener Arbeitsunfähigkeit: Wenn die Arbeitsunfähigkeit z.B. mobbingbedingt ist, sollte angegeben werden, ob und inwiefern der Patient in einem anderen personellen Umfeld beim Arbeitgeber beschäftigt werden kann [11]. Auch hier können Erkundigungen beim Arbeitgeber sinnvoll sein, was aber erneut die Zustimmung des Arbeitnehmers voraussetzt.

Aus ärztlicher Sicht liegt der Vorteil solcher situativ erweiterten Zeugnisse zunächst darin, dass der Arzt dadurch dokumentiert, dass er sorgfältig gehandelt, seiner Diagnose eigene Untersuchungen zugrunde gelegt und eine differenzierte Beurteilung vorgenommen hat. Damit beugt er auch dem Vorwurf vor, er habe ein unrichtiges Zeugnis ausgestellt. Schliesslich ist es auch weniger wahrscheinlich, dass er als Zeuge in einem Zivilverfahren oder gar als Angeschuldigter in einem Strafverfahren auftreten muss. Denn die Praxis zeigt, dass ausführlichere Arztzeugnisse bei Arbeitgebern auf deutlich grössere Akzeptanz stossen als wenig bis nichtssagende Kurzbescheinigungen. Aus den gleichen Überlegungen liegt es auch im Interesse des Patienten bzw. Arbeitnehmers, ein etwas aussagekräftigeres Zeugnis vorlegen zu können, da er dadurch dem latenten Verdacht, gar nicht arbeitsunfähig zu sein, zu einem guten Teil den Wind aus den Segeln nehmen kann [12]. Man könnte sich fragen, ob der Arzt auftragsrechtlich nicht sogar verpflichtet ist, den Patienten über die Vorteile eines aussagekräftigeren Zeugnisses aufzuklären.

Eine Klarstellung ist notwendig: Die vorstehenden Ausführungen wollen nicht so verstanden sein, dass allein aus Furcht vor hypothetisch drohenden Rechtshändeln nur noch Zeugnisse in Überlängen ausgestellt werden. In den meisten Fällen wird der zuvor geschilderte Mindestinhalt genügen. Dort aber, wo die erwähnten besonderen Umstände vorliegen, insbesondere bei objektiv kaum nachprüfbaren Befunden, bei rückwirkenden Attesten oder bei rein arbeitsplatzbezogenen Arbeitsunfähigkeiten, sind die empfohlenen Ergänzungen sinnvoll. 


\section{Abschliessende Empfehlungen}

Neben dem vorstehenden Plädoyer für situativ aussagekräftigere Zeugnisse ist aus anwaltlicher Sicht schliesslich die Befolgung folgender Empfehlungen ratsam:

- Es ist auf das Führen einer vollständigen und präzisen Krankengeschichte zu achten. Insbesondere sollten die vorgenommenen Untersuchungen und erhobenen Befunde dokumentiert werden. Die Krankengeschichte kann für den Arzt im Ernstfall, z.B. wenn gegen ihn ein Strafverfahren wegen falschen ärztlichen Zeugnisses eröffnet wird, zur veritablen «Lebensversicherung» werden. Denn mit Hilfe einer sorgfältig dokumentierten Krankengeschichte wird er den Vorwurf einer unsorgfältigen oder gar unterlassenen Untersuchung meist rasch entkräften können.

- Grosse Zurückhaltung ist bei der Auskunftserteilung an den nachfragenden Arbeitgeber oder im kollegialen Gespräch mit dem Vertrauensarzt angezeigt. Es gilt die strikte Regel: keine Kommunikation ohne Entbindung vom Berufsgeheimnis durch den Patienten [13]! Vor allem bei telefonischen Anfragen stellt sich das Zusatzproblem der Identifikation der anfragenden Person, d.h. ob es sich bei ihr tatsächlich um einen legitimierten Vertreter des Arbeitgebers handelt. Sofern hier Zweifel bestehen, ist es geboten, Sicherheitsvorkehrungen zu treffen. So kann das Telefonat zunächst höflich abgebrochen werden, um den Anrufer anhand der angegebenen Telefonnummer vor dem Rückruf zu überprüfen. Am besten wäre es ohnehin, wenn der Patient die auskunftsberechtigte Person mit Namen und nicht einfach nur mit «Arbeitgeber» bezeichnet [14]. Die Entbindungserklärung sollte ferner für den Konfliktfall eigentlich immer beweisbar vorgelegt werden können. Dies würde streng genommen stets eine formelle Bestätigung des Patienten (z.B. schriftlich oder per E-Mail) bedingen, was aber von diesem als überzogene Massnahme oder gar Ausdruck des Misstrauens ihm gegenüber verstanden werden kann. Ein pragmatischer Ansatz kann sein, die Entbindung mündlich in der Sprechstunde vom Patienten einzuholen und in der Krankengeschichte eine entsprechende Aktennotiz anzufertigen.

- Trotz all dieser Vorkehrungen können Ärzte in Zivil- oder gar Strafverfahren involviert werden. Dass ein Arzt in einem arbeitsrechtlichen Zivilverfahren als Zeuge angerufen wird, ist heute gang und gäbe und braucht als solches nicht zu beunruhigen. Auch den gelegentlich vorkommenden Provokationen durch Parteianwälte, sei es im Rahmen vorprozessualer Korrespondenzen (Beispiel aus einem Anwaltsbrief: «Als einfacher Hausarzt sind Sie absolut unfähig, eine Depression zu erkennen») oder bei der Befragung im Zeugenstand, wird am besten mit souveräner Gelassen- heit begegnet. Delikater ist die Situation, wenn der Arzt selber in den Fokus einer Strafuntersuchung gerät. Auch hier gilt es, Ruhe zu bewahren. Die Eröffnung einer Strafuntersuchung muss nicht viel bedeuten, wie das im ersten Teil erwähnte Fallbeispiel belegt. Dennoch ist es empfehlenswert, sich frühzeitig einen Überblick über die zur Verfügung stehenden Verfahrensrechte zu verschaffen und allenfalls rechtliche Unterstützung beizuziehen [15]. Als erste Anlaufstelle bieten sich die Ärzteverbände mit ihren Rechtsdiensten oder allenfalls abgeschlossene Rechtsschutzversicherungen an. Diese Stellen können auch bei der Entscheidung helfen, ob der Beizug eines Anwalts notwendig ist, und nötigenfalls entsprechende Kontakte vermitteln.

\section{Literatur}

1 Art. 324a und Art. 336c OR.

2 Vgl. die einleitenden Anmerkungen von Hanspeter Kuhn, Seite 912 oben.

3 Art. 34 der Standesordnung der FMH verlangt letzteres ausdrücklich.

4 Nicht zu verwechseln mit rückdatierten Zeugnissen, wenn also der Arzt in Bezug auf das Ausstellungsdatum des Zeugnisses ein falsches Datum einträgt. Solche Rückdatierungen sind in jedem Fall unzulässig.

5 Abrufbar via www.aerzte-zh.ch.

6 Streiff U, von Kaenel A. Arbeitsvertrag. 6. Auflage, Zürich, Basel, Genf; 2006, N 12 zu Art. 324a/b OR, mit weiteren Hinweisen auf die Rechtsprechung.

7 Er kann aber die Offenlegung der Diagnose ohne weiteres bewilligen.

8 Ebenso Müller AR: Arztzeugnisse in arbeitsrechtlichen Streitigkeiten. AJZ 2/2010. S. 168.

9 So auch die Empfehlungen der Ärzte-Gesellschaft des Kantons Zürich.

10 www.saez.ch $\rightarrow$ Archiv $\rightarrow 2010 \rightarrow 22$

11 Egli HP. Der Beweis der Arbeitsunfähigkeit Probleme mit Arztzeugnissen. In: Entscheide des Arbeitsgerichts Zürich. 2008. S. 49 f.

12 Zum Ganzen und weiterführend, insbesondere zu beweisrechtlichen Fragen: Kälin O. Das Arztzeugnis als Beweismittel bei arbeitsrechtlichen Streitigkeiten. ZZZ, 2006; S. 340-1.

13 Eine Ausnahme kann höchstens darin gesehen werden, dass der Arzt gegenüber dem zweifelsfrei identifizierten Arbeitgeber jene Angaben bestätigen darf, die bereits aus dem Zeugnis hervorgehen. Denn insofern offenbart der Arzt nicht mehr, als dem Arbeitgeber durch die Vorlage des Zeugnisses bereits bekannt ist. Allerdings: Allein deswegen, um das Zeugnis zu bestätigen, wird kaum ein Arbeitgeber den Kontakt mit dem Arzt suchen. Auch hier ist also Vorsicht angebracht.

14 Müller RA. a. a. O. S. 170.

15 Dazu der Beitrag von Tanja Knodel. Strafuntersuchung gegen Ärzte - wie sollen sich Betroffene verhalten? Schweiz Ärztezeitung. 2009(90)46:1804-5. 